\title{
Actinomycotic Endomyometritis Associated with a Long-Term Use of Intrauterine Device Lasting for 42 Years
}

\author{
Vladimír Bartoš ${ }^{1, *}$, Jana Doboszován ${ }^{1}$ Martin Sudek²
}

\section{ABSTRACT}

In women, pelvic actinomycosis is closely associated with prolonged use of the intrauterine devices (IUD). A 70-year old female presented with intermittent blood-stained vaginal discharge. An analysis of her history revealed, she was inserted with an IUD 42 years ago, but it has remained in situ untill now. Curettage of the uterus was done, but an IUD was firmly attached inside the cavity and there was not able to remove it. A biopsy material consisted of the large round and oval granules of filamentous and mycelium-like microorganisms. They showed strong positivity with Periodic acid-Schiff stain and Gömöri methenamine silver stain. Histopathology was consisted with uterine actinomycosis. A total abdominal hysterectomy with bilateral adnexectomy was performed. The uterus contained a retained plastic IUD. Microscopic investigation revealed a diffuse chronic active endomyometritis with sporadic Actinomycetes colonies. Wearing an IUD continuously for very long periods of time can lead to actinomycotic infection, which may manifest for many years after its application. All IUD users have to keep in mind regular gynecological check-ups to avoid the complications of a retained and "forgotten" IUD.

\section{KEYWORDS}

actinomycosis; endomyometritis; intrauterine device

AUTHOR AFFILIATIONS

${ }^{1}$ Department of Pathology, Faculty Hospital in Žilina, Slovakia

2 Department of Gynecology and Obstetrics, Faculty Hospital in Žilina, Slovakia

* Corresponding author: Faculty Hospital in Žilina, Vojtecha Spanyola 43, 012 07, Žilina, Slovakia; e-mail: vladim.bartos@gmail.com

Received: 2 October 2018

Accepted: 9 January 2019

Published online: 1 April 2019

Acta Medica (Hradec Králové) 2019; 62(1): 35-38

https://doi.org/10.14712/18059694.2019.44

(c) 2019 The Authors. This is an open-access article distributed under the terms of the Creative Commons Attribution License (http://creativecommons.org/licenses/by/4.0), which permits unrestricted use, distribution, and reproduction in any medium, provided the original author and source are credited. 


\section{INTRODUCTION}

Actinomycosis is a chronic suppurative disease caused by Actinomyces species. They represent Gram-positive, anaerobic or microaerophilic bacteria with a tendency to form branching filaments that fragment into the rods $(1,2)$. They are commensals normally encountered in the oral cavity, gastrointestinal tract, and female genital tract $(1,2)$. Under normal circumstances, Actinomycetes are unable to cross the mucosal barrier and cause disease. The main predisposing factor for acquiring an infection is an irritation of the mucosa by trauma or foreign bodies, chronic inflammatory disease, and immunosuppression $(1,2)$. These pathologic conditions allow bacterial entry into the deep tissue through damaged mucosal barrier (1). Actinomycosis exhibits certain specific clinicopathologic characteristics. A densely fibrotic foci spreading in the connective tissue is one of the features. Advanced lesions are accompanied by abscesses formation, which may lead to the development of sinuses and fistulas to adjacent organ structures (1). Histologically, actinomycosis is characterized by the finding of "sulfur granules", which are composed of the bacterial elements and tissue debris. In hematoxylin-eosin staining, "sulfur granules" are identified as round or oval basophilic masses with a radiating fringe of club-shaped configurations (Splendore-Hoeppli phenomenon) (1). The three main clinical forms of actinomycosis include cervicofacial, thoracic, and abdominopelvic. The last form accounts for approximately $20 \%$ of the cases (1). In women, pelvic actinomycosis is closely associated with prolonged use of the intrauterine devices (IUD) $(1,2)$. Herein, we present a unique case of endometrial actinomycosis associated with long-term insertion of an IUD in elderly woman.

\section{CASE PRESENTATION}

A 70-year old female who had been postmenopausal for 18 years, visited the Department of Gynecology (July, 2018) with intermittent vaginal discharge. It was brawnish in color and sometimes contained blood. The patient did not complain of pelvic pain nor had a higher body temperature. She had three spontaneous deliveries, the last one being 43 years previously (1975). No spontaneous abortion or medical interruption of pregnancy were recorded. A retrospective analysis of her history revealed that after the third birth, she was inserted (1976) with an IUD, that has remained in the uterus untill now. At present, she has been treated for diabetes mellitus type 2 with disease-related complications.

Per speculum and per vaginal examination did not show any pathologic lesion, that could be a source of discharge. Transvaginal ultrasonography (TVU) revealed a slightly enlarged uterine cavity filled with mixed, hyperechogenic and hypoechogenic masses. An IUD left in situ was clearly identify. The presumptive clinical diagnosis was an endometrial hyperplasia. The other findings of the TVU examination were within normal limits. Under general anesthesia, a curretage of the uterine cavity was done and a tissue specimen was sent for histopathology.

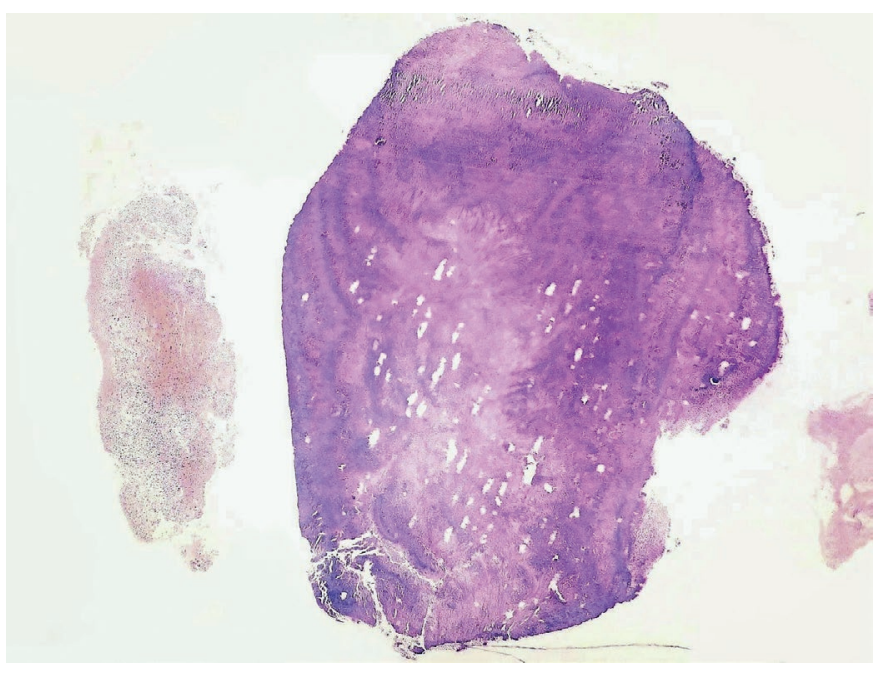

Fig. 1 High-power view of the basophilic masses of Actinomycetes (hematoxylin \& eozin, $\times 40$ ).

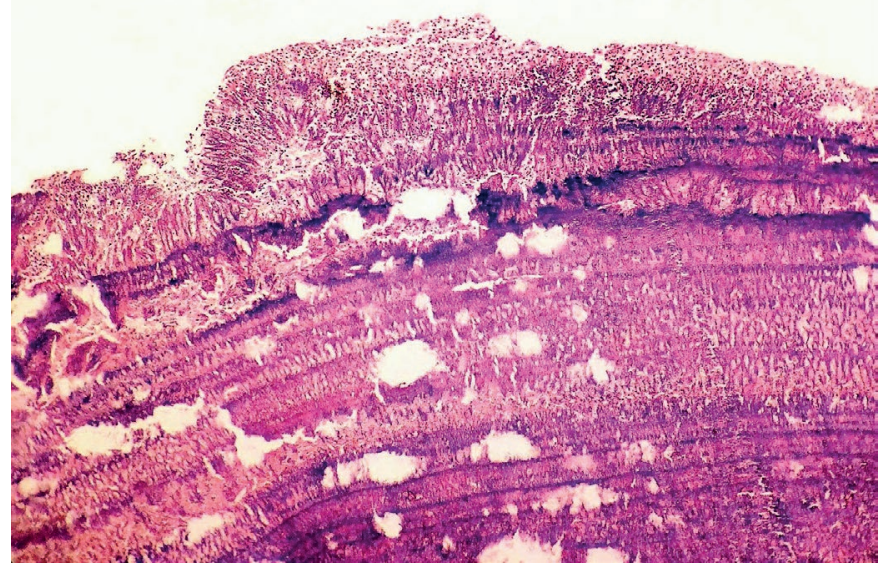

Fig. 2 Periodic acid-Schiff (PAS) stain of Actinomyces colonies. A narow rim of discrete leukocyte cellulisation at the periphery is visible $(\times 100)$.

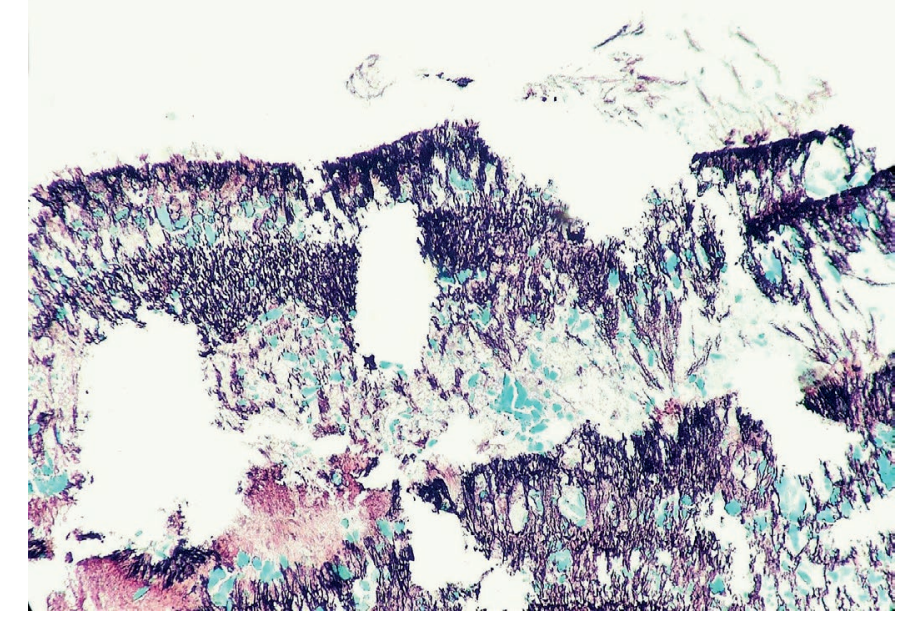

Fig. 3 Gömöri methenamine silver stain highlights abundant mycelia-like structures $(\times 100)$. 
However, an IUD was firmly attached inside the uterus and there was not able to remove it. Microscopically, a biopsy material consisted of the large (up to $5 \mathrm{~mm}$ in diameter) round and oval granules of basophilic microorganisms (Fig. 1). At the periphery, they were focally surrounded by thin layer of acute inflamatory infiltration. Splendore-Hoeppli phenomenon was sometimes seen. A high magnification revealed, these colonies were composed of branched filamentous and mycelium-like microorganisms situated in an amorphic matrix. They showed strong positivity with Periodic acid-Schiff (PAS) stain (Fig. 2) and Gömöri methenamine silver stain (Fig. 3). The rest of the biopsy specimen included necrotic inflamatory debris, fragments of heavily inflamed endometrium and blood content. Histopathology was consisted with uterine actinomycosis associated with prolonged use of IUD.

After receiving a biopsy report, the patient was consulted with the specialists to decide the further steps. Considering her age and particularly an inability to extract an IUD from the uterus, a total abdominal hysterectomy with bilateral adnexectomy was done a few weeks later. Grossly, the uterus measured $9 \times 5 \times 3 \mathrm{~cm}$ and contained retained plastic IUD inside the cavity (Fig. 4). An adjacent corporal endometrium appeared thin and hemorrhagic without any marked pathologic foci. Microscopic investigation of the samples revealed a finding of diffuse chronic active endomyometritis. Endometrium was atrophic and massively infiltrated by lymphocytes, plasma cells and leucocytes (Fig. 5), which affected an adjacent myometrium as well. In some areas, endometrium was totally replaced by nonspecific inflammatory granulation tissue. Only in a single excision, the colonies of actinomycotic microorganisms

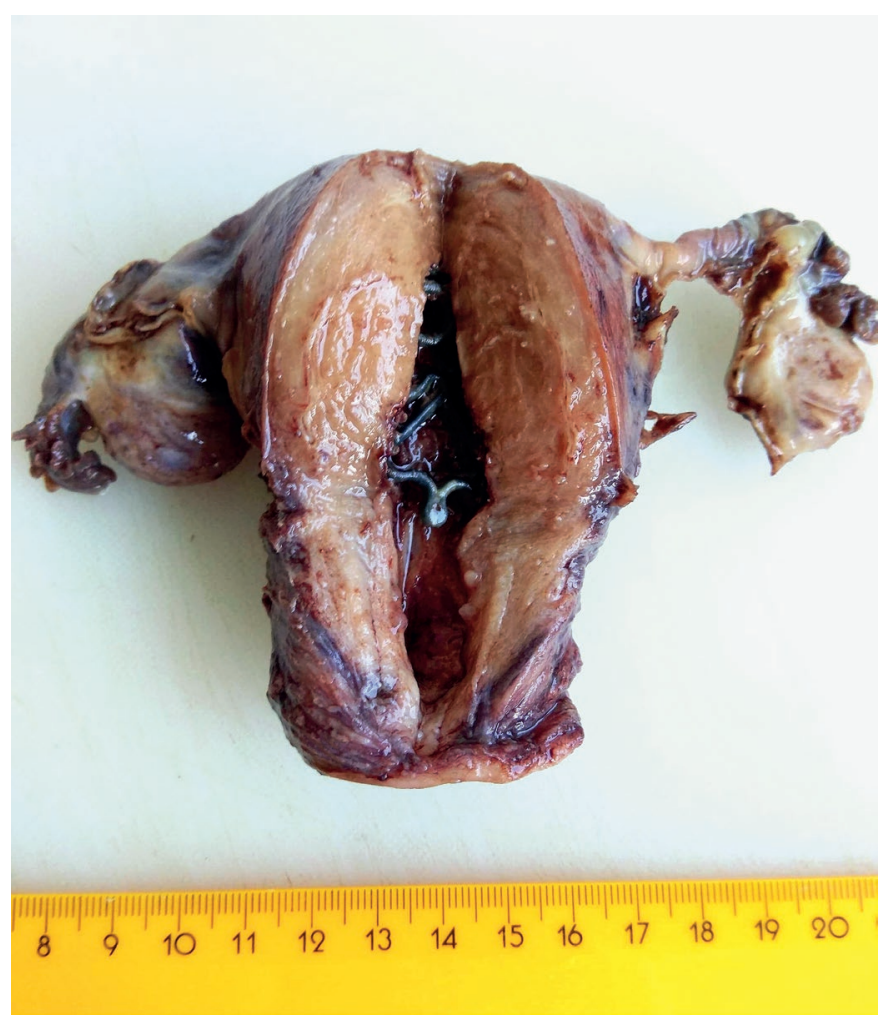

Fig. 4 Uterus with adnexa after hysterectomy (post fixation in formalin). An IUD left in situ is seen inside the cavity.

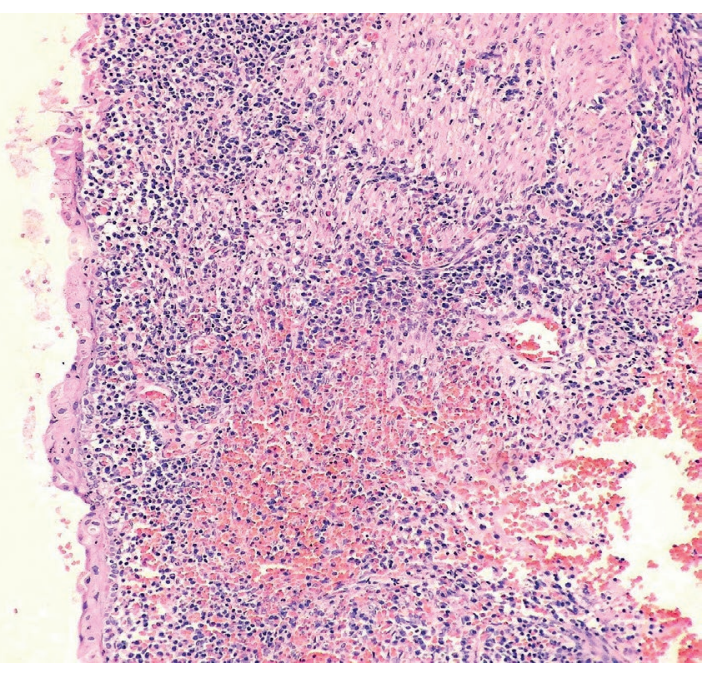

Fig. 5 Detail on massive inflammation of the atrophic endometrium and adjacent myometrium (hematoxylin \& eozin, $\times 100$ ).

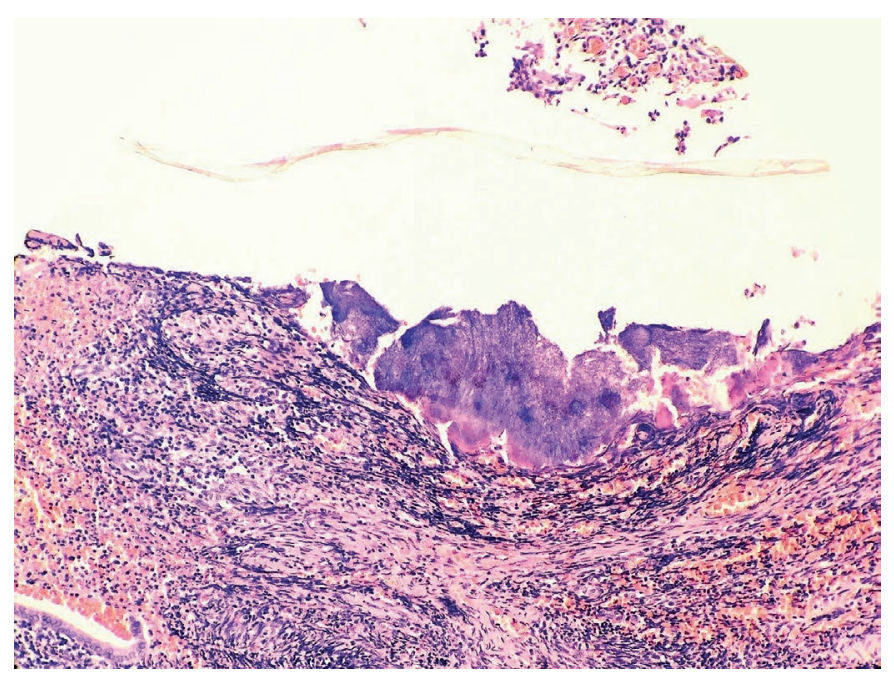

Fig. 6 Chronic active endomyometritis with Actinomyces colonies in the centre (hematoxylin \& eozin, $\times 40$ ).

were seen on the surface of almost entirely lost endometrium (Fig. 6). Both ovaries and fallopian tubes were normal without any signs of infection. The postoperative period was uneventful, the patient was administered with oral antibiotic therapy (Bitamon), and she was discharged from the hospital four days after surgery.

\section{DISCUSSION}

At the present time, actinomycosis of the uterus is an extremely rare disease. In a recent study, Chiesa-Vottero (3) has found only seven cases $(0.02 \%)$ of actinomycotic endometritis among 28,906 endometrial biopsies. Actinomycosis of the female genital tract is directly related to long-term use of the IUDs, especially for more than 5 years $(1,2)$. Prolonged insertion of an IUD causes mild inflammatory changes in the endometrium with necrosis that creates an anaerobic environment that favors the growth of the Actinomycetes (2). Although this association 
Tab. 1 Summary of the clinical findings of the patients extracted from the published case reports.

\begin{tabular}{|l|l|l|l|}
\hline Ref. & Patient age & IUD use & Clinical manifestation \\
\hline$[2]$ & 76 years & 44 years & $\begin{array}{l}\text { vaginal bleeding, pelvic } \\
\text { pain, mild febrility }\end{array}$ \\
\hline$[6]$ & 58 years & 33 years & $\begin{array}{l}\text { vaginal bleeding } \\
\text { and spotting }\end{array}$ \\
\hline$[7]$ & 67 years & 35 years & $\begin{array}{l}\text { acute abdominal pain, } \\
\text { perforation of the uterus }\end{array}$ \\
\hline$[8]$ & 55 years & 27 years & $\begin{array}{l}\text { vaginal bleeding } \\
\text { and spotting }\end{array}$ \\
\hline$[9]$ & 59 years & 22 years & $\begin{array}{l}\text { blood-stained vaginal } \\
\text { discharge, mild febrility }\end{array}$ \\
\hline this case & 70 years & 42 years & $\begin{array}{l}\text { blood-stained vaginal } \\
\text { discharge }\end{array}$ \\
\hline
\end{tabular}

is generally well-known, it is not possible to quantify an actual risk of disease. Approximately 7-13.7\% of women using an IUD may have a finding of Actinomyces-like microorganisms on a Papanicolaou test $(4,5)$. However, the prognostic significance of this finding is minimal because the vast majority of them will remain without any signs of disease $(4,5)$. For example, in the study of Kalaichelvan et al. (5), 150 out of 152 IUD users (98.7\%) with Actinomyces-positive cervical smears were asymptomatic, while only two $(1.3 \%)$ developed a pelvic inflammatory disease at 6 months. Therefore, in the absence of clinical symptoms, women with Actinomyces-like organisms on a Papanicolaou test do not need preemptive antibiotic treatment, even do not require IUD removal $(4,5)$. However, because one can not predict which IUD user colonized by Actinomyces will develop severe pelvic infection, all of them should have a periodical gynecological follow-up.

In the current paper, we have presented a rare case of postmenopausal woman with an actinomycotic endomyometritis associated with a long-term use of an IUD lasting for 42 years. To our knowledge, only a few articles desribing an endometrial actinomycosis related to such a long interval of an IUD insertion has been published until now. We have reviewed five such cases, which are briefly summarized in Table 1 (references 2, 6-9) including our present case. All females were postmenopausal with the mean age of 63 years. The time of an IUD insertion ranged from 22 to 44 years. The main clinical symptoms were vaginal discharge and bleeding. A very uncommon case has been published by Phupong et al. (7), who reported a 67-yearold woman manifesting with an acute abdominal pain due to spontaneous perforation of the uterus from actinomycotic infection with Lippes loop IUD.

In conclusion, wearing an IUD continuously for very long periods of time can lead to actinomycotic infection, which may manifest for many years after its application. As this method of contraception is being used much less frequently than in the past, such cases as we have described become a rarity. Anyway, all IUD users have to keep in mind regular gynecological check-ups to avoid the complications of a retained and "forgotten" IUD.

\section{ACKNOWLEDGEMENTS}

The authors would like to thank all physicians from Faculty Hospital in Žilina, who participated on treatment and clinical management of the patient.

\section{REFERENCES}

1. Heo SH, Shin SS, Kim JW, et al. Imaging of actinomycosis in various organs: a comprehensive review. Radiographics 2014; 34: 19-33.

2. Prabhala S, Erukkambattu J, Basavanapalli M, Tanikella R. Endometrial actinomycosis associated with intrauterine contraceptive device forgotten for 44 years. Med J DY Patil Univ 2016; 9: 231-3.

3. Chiesa-Vottero AG. Actinomycotic endometritis. Int J Gynecol Pathol 2019; 38(2): 138-42.

4. Westhoff C. IUDs and colonization or infection with Actinomyces. Contraception 2007; 75(6 Suppl): S48-50.

5. Kalaichelvan V, Maw AA, Singh K. Actinomyces in cervical smears of women using the intrauterine device in Singapore. Contraception 2006; 73: 352-5.

6. Sozen I, Morgan K, Shannon JS. Postmenopausal bleeding secondary to a Dalkon Shield retained for 33 years: a case report. J Reprod Med 2005; 50: 216-8.

7. Phupong V, Sueblinvong T, Pruksananonda K, Taneepanichskul S, Triratanachat S. Uterine perforation with Lippes loop intrauterine device-associated actinomycosis: a case report and review of the literature. Contraception 2000; 61:347-50.

8. Shweta N, Nandini JU, Laxmi PV. Pelvic actinomycosis associated with long term use of intrauterine contraceptive device. J Obstet Gynecol India 2010; 60: 345-7.

9. Kriplani A, Buckshee K, Relan S, Kapila K. "Forgotten" intrauterine device leading to actinomycotic pyometra 13 years after menopause. Eur J Obstet Gynecol Reprod Biol 1994; 53: 215-6. 